\title{
DESARROLLO DE GEMELOS DIGITALES PARA LA SIMULACIÓN E INTEGRACIÓN DE ACTIVOS DE FABRICACIÓN EN LA INDUSTRIA 4.0
}

\author{
D. Orive*, A. López*, E. Estévez**, A. Orive*, M. Marcos* \\ *Dept. Ingeniería de Sistemas y Automática, UPV/EHU, España \\ e-mail: \{dario.orive, alejandro.lopez, marga.marcos\}@ehu.eus, aorive005@ikasle.ehu.eus \\ **Dept. Ingeniería Electrónica y Automática EPS de Jaén, España \\ e-mail: eestevez@ujaen.es
}

\section{Resumen (12 ptos, negrita)}

El paradigma de la Industria 4.0 plantea el uso de herramientas y tecnologías que aumenten la eficiencia y la capacidad de adaptación de los procesos productivos para hacer frente a un mercado globalizado y con una alta competitividad. El concepto de Gemelo Digital es clave en el contexto de la Industria 4.0, y ha sido objeto de interés para la industria y la academia. Sin embargo, no hay consenso en la definición de este concepto, existiendo principalmente tres interpretaciones: la primera define un Gemelo Digital como un modelo de simulación de alta precisión; la segunda lo interpreta como una representación virtual del estado y el comportamiento de un activo de fabricación para su integración en la Industria 4.0; y la tercera, de reciente aparición, plantea la combinación de las anteriores. En este trabajo, se propone una metodología para la implementación de Gemelos Digitales que se ajusten a esta tercera interpretación. De esta forma, se proponen acciones concretas para la implementación de un Gemelo Digital que tenga capacidad de simular una estación y realizar su puesta en marcha virtual, además de facilitar su integración en la Industria 4.0.

Palabras clave: Gemelo Digital, Industria 4.0, Puesta en Marcha Virtual, Sistemas de Fabricación Flexibles.

\section{INTRODUCCIÓN}

El paradigma de la cuarta revolución industrial, también denominada Industria 4.0, surge como respuesta ante la transformación hacia un mercado globalizado y con una alta competitividad. En un contexto en el que el cliente exige cada vez una mayor calidad y personalización de los productos, la Industria 4.0 plantea el uso de herramientas y tecnologías que aumenten la eficiencia y la capacidad de adaptación de los procesos productivos [6]. La Industria 4.0 basa el cumplimiento de estos objetivos en el valor añadido de los datos que se generan en planta. De esta manera, propone el uso de una serie de tecnologías dedicadas a la adquisición y el procesamiento de datos y la posterior realimentación de acciones inferidas de ese análisis (inteligencia artificial, big data, simulación, sistemas ciber-físicos, internet de las cosas, etc.) [12].

Uno de los conceptos que mayor repercusión e impacto ha tenido en el contexto de la Industria 4.0 es el de Gemelo Digital, recibiendo gran atención durante los últimos años tanto por parte de la academia [1],[9] como de la industria [7], [5]. Este concepto consiste en una representación virtual de la realidad a lo largo de las diferentes fases del ciclo de vida de un producto, desde la concepción o idea inicial, pasando por los diferentes procesos de fabricación, trazabilidad, distribución y venta, hasta el reciclado final del mismo [4]. Sin embargo, no hay un consenso respecto al alcance y las funcionalidades que debe tener un Gemelo Digital.

Una corriente de pensamiento está alineada con la primera definición que se le dio al término Gemelo Digital en el año 2003, según la cual, un Gemelo Digital es un modelo de simulación de alta precisión utilizado para el análisis y optimización de procesos de fabricación [3]. Los autores alineados con esta postura defienden que las interfaces de entrada y salida de datos de un Gemelo Digital deben ser las mismas que las del proceso que replican, de forma que para el controlador la interacción con el proceso real y con su Gemelo Digital sea idéntica [1].

Además, revisiones más actualizadas de esta idea mantienen que los Gemelos Digitales deben ser de utilidad no solo para simulación offline, sino que se deben de utilizar de forma dinámica, utilizando la información adquirida del proceso real para fines como la adaptación de parámetros o el mantenimiento predictivo [10], incluso para llevar a cabo la puesta en marcha virtual del proceso, lo que permite reducir tiempo, recursos y costes a la hora de poner en marcha procesos o máquinas reales [7]. Esto implica que el Gemelo Digital no solo debe presentar las mismas interfaces de entrada y salida que el proceso real, sino 
que, además, permita validar las soluciones de control y automatización a implementar en el proceso real.

Por otra parte, diferentes autores y organizaciones, como la plataforma alemana para la Industria 4.0, definen un Gemelo Digital como una representación digital de las características y los comportamientos/funcionalidades de una entidad de fábrica, generalmente de un activo de planta (p.ej., un robot industrial) [2]. Esta aproximación está muy ligada al concepto de los sistemas ciber-físicos, formado por una parte física (un activo de planta), y una parte virtual (es decir, su Gemelo Digital) que representa el estado de la parte física, permitiendo así su interacción con otros activos en un intercambio de información y servicios [13]. De esta manera, el Gemelo Digital se convierte en imprescindible para la integración de activos de fabricación dentro de arquitecturas para la Industria 4.0 [5].

Sin embargo, en los últimos años ha surgido una tercera vía, que plantea que ambas aproximaciones no solo son compatibles, sino que deberían ser integradas en un único concepto más amplio de Gemelo Digital [8]. De esta manera, se interpreta al Gemelo Digital como una entidad encargada de gestionar al activo físico, representando su estado y sus funcionalidades en arquitecturas 4.0, e integrando las capacidades de simulación y puesta en marcha virtual como recursos con los que poder ejecutar las decisiones resultantes de esta gestión [11]. Para ello, se considera que para cumplir con este objetivo un Gemelo Digital debe permitir lo siguiente:

- Definir y simular trayectorias, considerando el activo y su entorno.

- Definir y simular el control de la estación, y generar el código para la estación real.

- Definir y simular la automatización de la estación, y generar el código para la estación real.

- Definir y simular las capacidades de comunicación y procesamiento de datos para su participación en el intercambio de información y servicios con otros Gemelos Digitales.

En este trabajo se propone una metodología para la implementación de Gemelos Digitales que se ajusten a este concepto en el que se integran las capacidades de simulación y gestión de activos.

El resto del artículo está organizado de la siguiente manera: el apartado 2 describe las herramientas para el desarrollo de gemelos digitales y su integración en la Industria 4.0; el apartado 3 presenta un caso de estudio; finalmente, el apartado 4 cierra el trabajo con las conclusiones de los autores.

\section{SIMULACIÓN E INTEGRAGIÓN DE ACTIVOS INDUSTRIALES}

En la sección anterior, se ha comprobado que el concepto de Gemelo Digital ha sido interpretado de múltiples maneras en la literatura y la industria, dando lugar a diferentes aproximaciones. La implementación final de un Gemelo Digital, así como el conjunto de herramientas utilizadas para su desarrollo, varían en gran medida dependiendo de los objetivos que se persiguen con el mismo.

Una vez definido el concepto de Gemelo Digital que se desea implementar, en primer lugar hay que definir las pautas para poder desarrollarlo. El siguiente paso consistirá en la elección de herramientas adecuadas para implementar el Gemelo Digital deseado, atendiendo a la integridad de la solución. Finalmente, se concreta cómo llevar a cabo las pautas propuestas pautas para el desarrollo de Gemelos Digitales utilizando las herramientas seleccionadas.

\subsection{ACCIONES PARA EL DESARROLLO DEL GEMELO DIGITAL}

La implementación de un Gemelo Digital puede resultar compleja, debido a la diversidad de funcionalidades que debe soportar para cumplir con los requisitos que se han identificado en la introducción. Para simplificar esa tarea, los autores proponen dividir la implementación en una serie de acciones concretas:

- Modelado de la estación: el primer paso consiste en la generación del modelo de simulación de la estación. De acuerdo con los requisitos que se han definido, este modelo tiene que permitir la generación de trayectorias y del código de control del activo.

- Proyecto de automatización: Una vez creado el modelo de simulación de la estación, el siguiente paso es generar un proyecto de automatización para el control de este. La lógica de este proyecto de automatización deberá ser válida para el Gemelo Digital o para el activo de fabricación real.

- Integración en arquitecturas para la Industria 4.0: Por último, hay que dotar al proyecto de automatización de recursos para procesar los datos que se reciben del proceso (sea el proceso real o el simulado), así como de la capacidad de compartir esos datos con otros activos de la fábrica mediante protocolos de comunicación de uso habitual en la Industria 4.0 (OPC UA, MQTT, etc.).

\subsection{ELECCIÓN DE HERRAMIENTAS}

Para poder poner en práctica las pautas del subapartado anterior, resulta necesaria la interacción y cooperación de diferentes herramientas software. Esta distribución permite un tratamiento individualizado de 
los diferentes requisitos, utilizando la herramienta apropiada en cada caso. Sin embargo, también implica que las diferentes herramientas utilizadas puedan trabajar de forma integrada, permitiendo simular las diferentes funcionalidades del Gemelo Digital de manera conjunta.

Teniendo en cuenta estos factores, se ha decidido utilizar herramientas de la plataforma PLM de Siemens como base para el concepto de Gemelo Digital propuesto por los autores. En concreto, se han considerado las siguientes herramientas (Figura 1):

- Tecnomatix Process Simulate (PS): permite el desarrollo del Gemelo Digital, la definición y simulación de trayectorias, así como la definición, generación y simulación del código de control. También facilita un API para la simulación con otras herramientas.

- TIA Portal: permite generar proyectos de automatización.

- PLCSIM Advanced: permite ejecutar en simulación el código de un proyecto de automatización desarrollado en TIA Portal.

- SIEMENS ODK 1500S: permite la invocación de funciones ejecutadas sobre un sistema operativo Windows desde un proyecto de automatización desarrollado en TIA Portal, permitiendo definir, generar y simular el código para hacer procesamiento y envío de datos hacia otros activos de fabricación.

\subsection{PARTICULARIZACIÓN DE LAS ACCIONES}

En este apartado se particularizan las pautas para implementar Gemelos Digitales definidas en el apartado 2.1 utilizando las herramientas definidas en el apartado 2.2

\subsubsection{Modelado de la estación}

PASO 1: Construcción del Gemelo Digital en Tecnomatix PS.

Una vez definida la estación de trabajo que se quiere desarrollar y el equipamiento que la compone, el primer paso para construir su Gemelo Digital es disponer de los modelos CAD de todos los componentes que la conforman.

Tras introducir el propio activo que realiza las operaciones, el siguiente elemento en orden de relevancia es la herramienta de trabajo del activo. Las herramientas requieren de un Punto Central de la Herramienta (o TCP), el cual determina el posicionamiento del activo a la hora de realizar operaciones.

Finalmente, se deben ubicar el resto de los elementos del escenario, definiendo las zonas de entrada, montaje y salida, así como los alimentadores de piezas.

Hay que indicar que la validación del escenario real a partir de lo definido en el Gemelo Digital no es algo inmediato ni fácil de conseguir. Durante el desarrollo del Gemelo Digital hay que hacer una serie de pruebas y comprobaciones de ciertos movimientos a puntos importantes del escenario que permitan asegurar la máxima similitud del Gemelo Digital respecto de la estación real. Si esto no se hace de una forma precisa, se presentarán problemas cuando lo desarrollado y validado en el Gemelo Digital se vuelque a los controladores de la estación real. A pesar ello, siempre habrá que hacer pequeños ajustes al pasar del mundo virtual al real, pero cuanto más precisa sea esta validación previa, menos ajustes habrá que realizar.

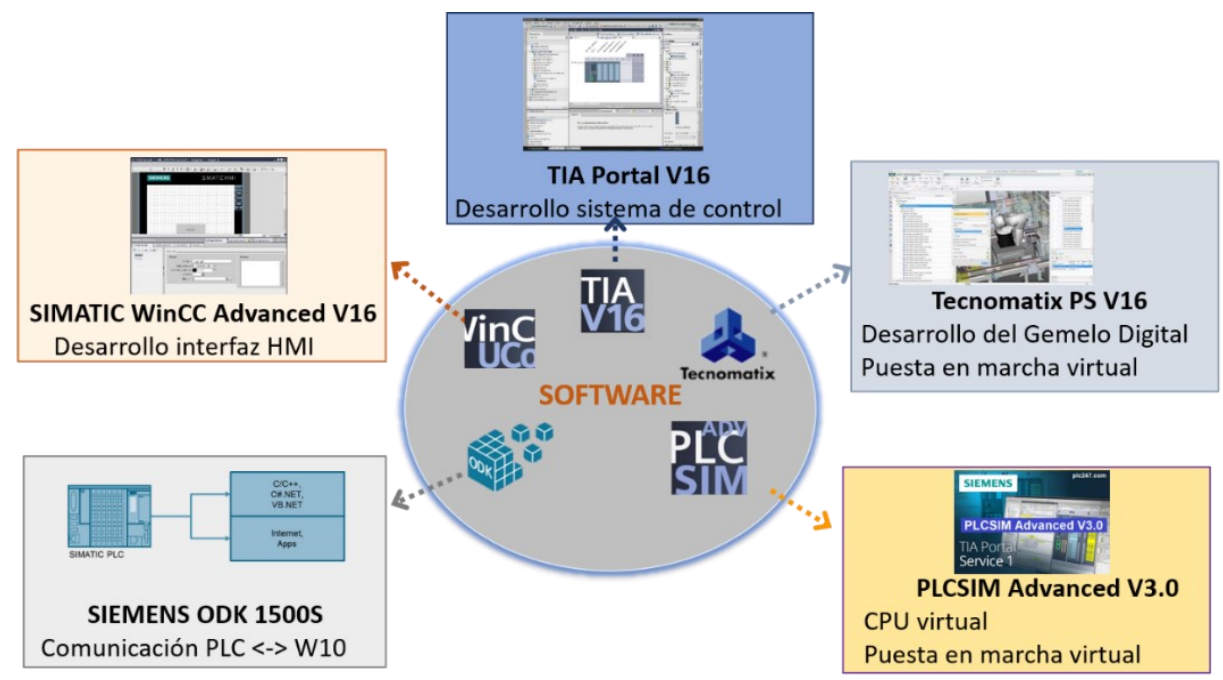

Figura 1: Integración de herramientas para el desarrollo de un Gemelo Digital 
PASO 2: Estructura del modelo del Gemelo Digital en Tecnomatix PS.

Después de introducir los componentes de la estación en el Gemelo Digital, hay que generar las trayectorias de las operaciones y el flujo de materiales.

Para la generación de trayectorias hay que definir qué tipo de CPU virtual se va a utilizar en la simulación del Gemelo Digital. Existe la opción de una CPU genérica, en cuyo caso el código generado para las trayectorias es específico de la herramienta Texnomatix PS. Como alternativa, se puede seleccionar la CPU virtual del activo que se esté utilizando en el Gemelo Digital, en cuyo caso, el código generado para las trayectorias se podrá utilizar en el control del activo en la estación real.

Por otra parte, para realizar simulaciones con el Gemelo Digital es necesaria la definición del flujo de materiales o Material Flow. En esta parte del modelado se definen los caminos que pueden seguir las piezas que se manipulan en el gemelo, durante la ejecución de los servicios.

\subsubsection{Proyecto de automatización del Gemelo Digital desde PLC}

PASO 3: Estructura del programa de automatización de la estación en TIA Portal.

Tras desarrollar el Gemelo Digital en Tecnomatix PS y validar el programa de control del activo en simulación, hay que realizar el programa de control del PLC que permita seleccionar en cada caso las operaciones a realizar en función del servicio solicitado a la estación. Este programa de control debe ser válido para la simulación con Software in the Loop (SiL) y para su ejecución en el PLC real en el control de la estación real. El proyecto de control de la estación se implementa en la herramienta de ingeniería TIA Portal, y para la simulación con el Gemelo Digital se ejecuta en la CPU virtual PLCSIM Advanced.

\subsubsection{Integración en arquitecturas para la Industria 4.0}

PASO 4: Comunicación PLC-W10, mediante SIEMENS ODK 1500S.

Como se ha explicado anteriormente, el uso de la librería SIEMENS ODK 1500S permite ejecutar desde un proyecto de automatización funciones que se ejecuten sobre un sistema operativo Windows. Esto permite la utilización de código desarrollado en lenguajes de alto nivel (C++, C\#, VB). Este tipo de funciones aportan una gran flexibilidad a la hora de procesar y enviar los datos con diferentes protocolos de comunicación. Por este motivo, se ha optado por esta herramienta para la publicación de datos.

\section{CASO DE ESTUDIO}

En esta sección se presenta un caso de estudio en el que se muestra cómo un Gemelo Digital desarrollado según los pasos propuestos por los autores se utiliza para realizar la puesta en marcha virtual de una estación de la que se recogen datos de proceso y se publican en una base de datos mediante el protocolo MQTT para su posterior análisis.

\subsection{DESCRIPCIÓN DE LA ESTACIÓN}

La estación de trabajo replicada en este caso de estudio mediante un Gemelo Digital ha sido diseñada para realizar el ensamblado de un conjunto de piezas, fabricadas mediante impresión 3D, que emulan un eje de motores paso a paso. Este conjunto se compone de cinco partes: base, rodamiento, bulón, tapa interior, y tapa exterior (Figura 2).

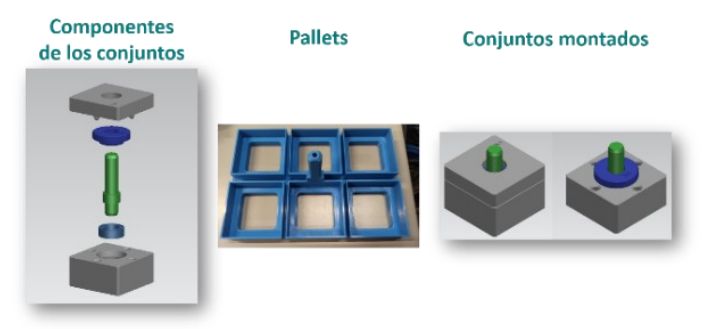

Figura 2: Detalle del producto fabricado en la estación del caso de uso

La estación recibirá peticiones de operaciones, o servicios, que pueden implicar el montaje total o parcial del conjunto. Cada servicio identifica una situación del producto a la entrada en la estación y a la situación a la salida de esta. Para el producto fabricado en esta estación, se han identificado 10 servicios que se indican en la tabla 1.

De cara a la ejecución de un servicio, la estación recibe pallets a su entrada con un número variable de ítems que puede oscilar entre una y seis piezas. El servicio se ejecuta para todos los conjuntos de un pallet o pedido, por lo que todos los conjuntos tienen que llegar en la misma situación.

Como se puede ver en la tabla 1, para el servicio número 4, en el pallet llega solo la pieza base del conjunto y se colocan en la estación el resto de los componentes, hasta finalizar el montaje. Sin embargo, en el servicio 6, en el pallet llegan conjuntos con la base y el rodamiento desde una estación previa y, se colocan el bulón y la tapa blanca, dejando los conjuntos en la zona de salida a falta de colocar la tapa exterior en otra estación. 
Tabla 1: Detalle de los servicios de fabricación ofrecidos por la estación

\begin{tabular}{|c|c|c|c|}
\hline Servicios & $\begin{array}{c}\text { Situación de } \\
\text { entrada }\end{array}$ & \multicolumn{2}{|c|}{ Tareas a realizar } \\
\hline 1 & \multirow{6}{*}{ Base } & Rodamiento & \\
\hline 2 & & Rodamiento & Bulón \\
\hline \multirow{2}{*}{3} & & Rodamiento & Bulón \\
\hline & & Tapa Interior & \\
\hline \multirow{2}{*}{4} & & Rodamiento & Bulón \\
\hline & & Tapa Interior & Tapa Exterior \\
\hline 5 & \multirow{4}{*}{$\begin{array}{c}\text { Base }+ \\
\text { Rodamiento }\end{array}$} & Bulón & \\
\hline 6 & & Bulón & Tapa Interior \\
\hline \multirow{2}{*}{7} & & Bulón & Tapa Interior \\
\hline & & Tapa Exterior & \\
\hline 8 & \multirow{2}{*}{$\begin{array}{c}\text { Base }+ \\
\text { Rodamiento } \\
+ \text { Bulón }\end{array}$} & Tapa Interior & \\
\hline 9 & & Tapa Interior & Tapa Exterior \\
\hline 10 & $\begin{array}{l}\text { Base }+ \\
\text { Rodamiento } \\
+ \text { Bulón }+ \\
\text { Tapa Interior }\end{array}$ & Tapa Exterior & \\
\hline
\end{tabular}

De cara a la ejecución de un servicio, la estación recibe pallets a su entrada con un número variable de ítems que puede oscilar entre una y seis conjuntos. El servicio se ejecuta para todos los conjuntos de un pallet o pedido, por lo que todos los conjuntos tienen que llegar en la misma situación a la estación.

Como se puede ver en la tabla 1, p.ej., para el servicio número 4 , en el pallet llega solo la pieza base del conjunto y se colocan en la estación el resto de los componentes, hasta finalizar el montaje. Sin embargo, en el servicio 6, en el pallet llegan conjuntos con la base y el rodamiento desde una estación previa y, se colocan el bulón y la tapa blanca, dejando los conjuntos en la zona de salida a falta de colocar la tapa exterior en otra estación.

Para realizar estos servicios de fabricación, la estación cuenta con un robot industrial KUKA KR3 R540 AGILUS, controlado mediante una controladora KR C4 COMPACT. Este robot está equipado con una herramienta terminal equipada con dos pinzas que permiten la manipulación de las distintas piezas del conjunto. Estas pinzas son controladas por el robot mediante un nodo EtherCAT con E/S digitales.

A su vez, el robot es controlado por un PLC Open Controller de Siemens, modelo ET200 SP PC2. Este equipo tiene la particularidad de estar equipado con un controlador de software PLC convencional, y tener además un sistema operativo Windows 10 embebido sobre el que se pueden desplegar funcionalidades con mayor capacidad de procesamiento.

El robot está ubicado sobre una mesa de aluminio con los dispositivos de maniobra y protección de las acometidas eléctrica y neumática. Sobre esta mesa, la estación cuenta con dos posiciones de entrada, por las que se reciben las materias primas (base y tapa exterior) y/o productos semielaborados sobre los que realizar un servicio de fabricación, y con una posición salida, donde se dejan los productos acabados o semielaborados después de realizar el servicio demandado. Además, la estación también cuenta con almacenes de materiales consumibles (rodamiento, bulón y tapa interior), necesarios para completar el ensamblado del conjunto (Figura 3).

\subsection{PUESTA EN MARCHA VIRTUAL CON SOFTWARE IN THE LOOP (SiL)}

Una vez desarrollado el Gemelo Digital de la estación y el programa de control de la misma para un PLC, el siguiente paso es realizar simulaciones entre ambas partes para validar las prestaciones de ambas partes en simulación, antes de utilizarlo en la estación real. Esta simulación se puede realizar con Software in the Loop (SiL) o con Hardware in the Loop (HiL). En este caso, se ha optado por hacerlo con (SiL).

En la simulación con SiL se lleva a cabo en una única plataforma PC en Windows 10, e intervienen varias herramientas software:
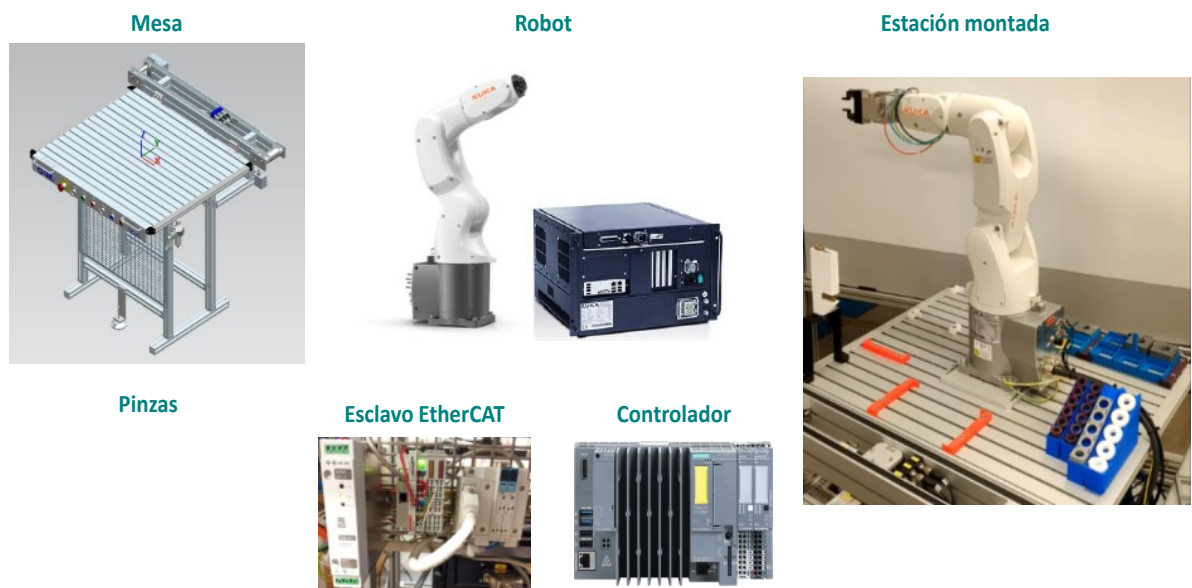

Figura 3: Detalle de los recursos utilizados en el caso de estudio 
- TIA Portal V16: Herramienta en la que se ha desarrollado el proyecto de control de la estación y que habrá que utilizar para observar y forzar valores de variables del proceso, así como para depurar el programa de control.

- PLCSIM Advanced V3.0: CPU virtual de PLC que permite ejecutar programas de PLC.

- Visual Studio: Herramienta en la que se ha desarrollado un programa que adapta los datos (en este caso, se transforma el formato de fechas recibido desde el PLC al formato esperado en la base de datos), y otro programa para la publicación de los datos generados en fabricación en la base de datos, utilizando la plataforma ODK de TIA Portal. La aplicación desarrollada en Visual Studio con ODK, genera 2 ficheros que hay que instalar en carpetas concretas de Windows 10. El fichero *.scl permite integrar las aplicaciones en el código de control. El fichero *.dll continene la aplicación ODK para comunicarse con el entorno Windows 10 , que se instalará en la instancia del PLC virtual de PLCSIM Advanced. El código de control se carga en el PLC virtual que, una vez mapeadas las E/S con Tecnomatix PS, permite llevar a cabo la puesta en marcha virtual.

- Tecnomatix PS: Por último, hay que poner en simulación el gemelo digital desarrollado en Tecnomatix PS. Hay que realizar el mapeado de E/S del PLC virtual con S/E del modelo en Tecnomatix PS (figura 4)

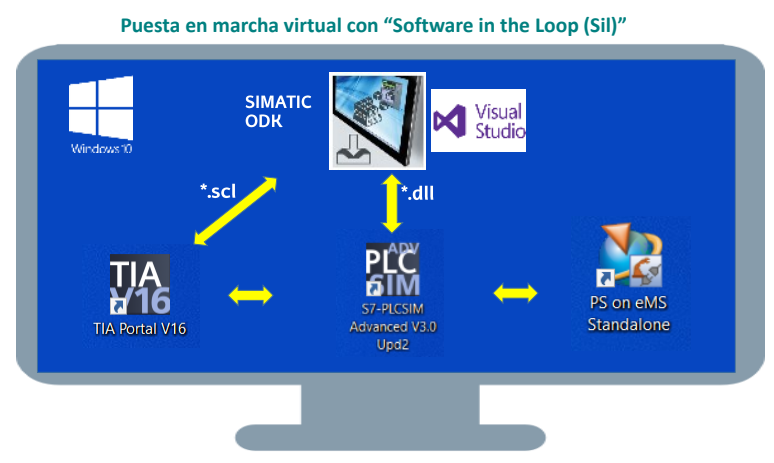

Figura 4: Detalle de la interacción entre las herramientas utilizadas para la puesta en marcha virtual

Durante esta fase se han ajustado valores y se ha depurado el sofware hasta que se ha validado el programa de control.

\subsection{PUESTA EN SERVICIO DE LA ESTACIÓN REAL}

Una vez validados los programas del robot y del PLC en simulación con SiL, la última operación que hay que realizar es poner en funcionamiento la estación real utilizando los proyectos utilizados en simulación. Esta operación se ha realizado en los siguientes pasos.

\section{PASO 1: Proyecto de control del robot Kuka.}

El proyecto de control de un robot Kuka consta de dos partes; la configuración del hardware y el software de la aplicación. Los programas de control de un robot Kuka están estructurados o divididos en 2 ficheros. El *.DAT que contiene la información sobre los puntos de las trayectorias y el *.SCR en el que se encuentran las instrucciones de movimiento del robot.

Indicar que, si en Tecnomatix PS se ha seleccionado la CPU del robot de Kuka, se generan estos dos ficheros en la fase de creación de las trayectorias del gemelo digital. Los ficheros así generados, se pueden utilizar en el robot real sin tener que realizar ninguna modificación.

\section{PASO 2: Montaje del sistema de control real.}

Para poner en servicio la estación real con el robot, es necesario disponer de un conjunto de dispositivos, un conjunto de herramientas software y la comunicación entre todos ellos (ver figura 5).

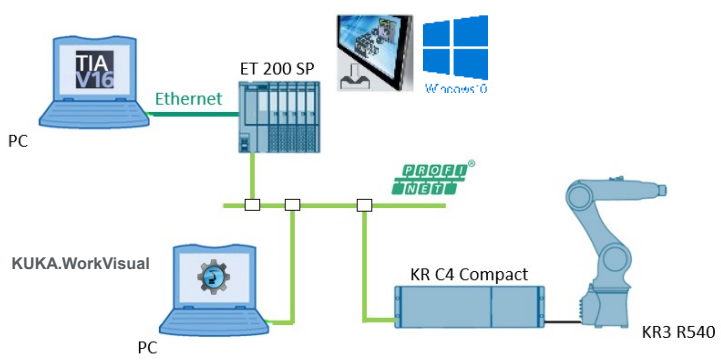

Figura 5: Sistema de control de la célula

En la Puesta en marcha real, el proyecto de control de PLC validado en la puesta en marcha virtual, se carga en el PLC ET 200 SP, en el cual se ha instalado previamente el fichero *.dll, en la parte de Windows 10, para la comunicación ODK. El PLC se comunica con la Unidad de Control del Robot, vía Profinet IO. El PLC tiene el rol de Controlado IO y el robot tiene rol de Dispositivo IO, en Profinet. Las señales que se intercambian entre ambos dispositivos se configuran en un proyecto con la herramienta de ingeniería KUKA.WorkVisual. Utilizando la herramienta KUKA.WorkVisual se han copiado en la Unidad de Control del Robot los ficheros *.DAT y *.SRC generados en simulación en Tecnomatix PS.

\section{CONCLUSIONES}

Este artículo se centra en el concepto de Gemelo Digital debido a su relevancia e impacto en el contexto 
de la Industria 4.0. Se revisan de forma breve las diferentes interpretaciones que recibe el término, resultando la más interesante para los autores aquella que plantea combinar las capacidades de simulación del activo con las de representación y gestión de este para integrarlo en arquitecturas para la Industria 4.0.

En este contexto, se ha realizado una propuesta para la implementación de Gemelos Digitales que sigan esta aproximación, para lo que se ha llevado a cabo un análisis de los pasos a realizar y las herramientas adecuadas para ello. En opinión de los autores, la principal ventaja del concepto propuesto es la facilidad de integración entre las diferentes herramientas necesarias para desarrollar el Gemelo Digital. Si bien es cierto que se trata de una solución ligada a un único fabricante, en este caso Siemens, se ha considerado que resulta de utilidad, dado que se trata de un fabricante con un gran peso específico en la industria.

\section{Agradecimientos}

Este trabajo está financiado por MCIU/AEI/FEDER, UE (proyecto RTI2018-096116-B-I00) y GV/EJ (proyecto IT1324-19).

\section{English summary}

\section{DEVELOPMENT OF DIGITAL TWINS TO SIMULATE AND INTEGRATE MANUFACTURING ASSETS IN THE INDUSTRY 4.0}

\begin{abstract}
The paradigm of Industry 4.0 proposes the use of technologies and tools to improve the efficiency and the adaptability of manufacturing processes to face a globalized and competitive market. The Digital Twin is a key concept within the context of Industry 4.0 and has received great attention from both industry and academia. However, there is a lack of consensus in the definition of this concept. There are mainly three interpretations: the first defines the Digital Twin as a high precision simulation model; the second interprets it as a virtual representation of the state and the behavior of manufacturing assets for its integration into Industry 4.0; and the third, of recent appearance, which proposes the combination of the previous ones. In this work, it is proposed a methodology to implement Digital Twins according to this third interpretation. Therefore, the authors propose concrete actions to implement a Digital Twin with the ability to simulate an station and perform its virtual commissioning, besides facilitating its integration in Industry 4.0.
\end{abstract}

Keywords: Digital Twin, Industry 4.0, Virtual Commissioning, Flexible Manufacturing Systems.

\section{Referencias}

[1] Gabor, T., Belzner, L., Kiermeier, M., Beck, M.T., Neitz, A., (2016) "A Simulation-Based Architecture for Smart Cyber-Physical Systems", 2016 IEEE International Conference on Autonomic Computing (ICAC), pp. 374-379.

[2] Glossary, disponible en https://www.plattformi40.de/PI40/Navigation/EN/Industrie40/Glossar y/glossary.html (visitado el 16/06/2021).

[3] Grieves, M., (2015) Digital Twin: Manufacturing Excellence through Virtual Factory Replication.

[4] Orive, D., Iriondo, N., Casquero, O., Marcos, M., (2019) "Hacia la puesta en marcha virtual de células robotizadas basada en gemelo digital", XL Jornadas de Automática, pp. 147-154.

[5] Platenius-Mohr, M., Malakuti, S., Grüner, S., Goldsch, T., (2019) "Interoperable Digital Twins in IIoT Systems by Transformation of Information Models: A Case Study with Asset Administration Shell", Proceedings of the 9th International Conference on the Internet of Things, pp 1-8.

[6] Santos, C., Mehrsai, A., Barros, A.C., Araújo, M., Ares, E., (2017) Towards Industry 4.0: an overview of European strategic roadmaps, Procedia Manufacturing.

[7] Schroeder, G., Steinmetz, C., Pereira, C.E., Muller, I., García, N., Espindola, D., Rodrigues, R., (2019) "Visualising the digital twin using web services and augmented reality", 2016 IEEE 14 th International Conference on Industrial Informatics (INDIN), pp 522-527.

[8] Tao, F., Qi, Q., Wang, L., Nee, A.Y.C., (2019) Digital Twins and Cyber-Physical Systems toward Smart Manufacturing and Industry 4.0: Correlation and Comparison, Engineering.

[9] Tao, F., Zhang, H., Liu, A., Nee, A.Y.C., (2019) Digital Twin in Industry: State-of-the-Art, IEEE Transactions on Industrial Informatics.

[10] Vachálek, J., Bartalský, L., Rovný, O., Šišmišová, D., Morháč, M., Lokšík, M., Hoffmeister, M., Zimermann, P., (2017) "The 
digital twin of an industrial production line within the industry 4.0 concept", 2017 21st International Conference on Process Control (PC), pp. 258-262.

[11] Wagner, C., Grothoff, J., Epple, U., Drath, R., Malakuti, S., Grüner, S., Hoffmeister, M., Zimermann, P., (2017) "The role of the Industry 4.0 asset administration shell and the digital twin during the life cycle of a plant", 201722 nd IEEE International Conference on Emerging Technologies and Factory Automation (ETFA), pp. 1-8.

[12] Wang, S., Wan, J., Li, D., Zhang, C., (2016) Implementing Smart Factory of Industrie 4.0: An Outlook, International Journal of Distributed Sensor Networks.
[13] Weyer, S., Meyer, T., Ohmer, M., Gorecky, D., Zühlke, D., (2016) Future Modeling and Simulation of CPS-based Factories: an Example from the Automotive Industry, IFACPapersOnLine.

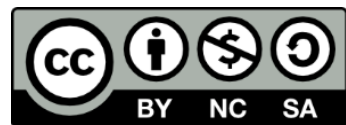

(C) 2021 by the authors. Submitted for possible open access publication under the terms and conditions of the Creative Commons Attribution CC BY-NC-SA 4.0 license (https://creativecommons.org/licenses/byncsa/4.0/deed.es). 